\title{
A Perching Mechanism for Flying Robots Using a Fibre-Based Adhesive
}

\author{
Ludovic Daler ${ }^{1}$, Adam Klaptocz ${ }^{1}$, Adrien Briod ${ }^{1}$, Metin Sitti $^{2}$ and Dario Floreano ${ }^{1}$
}

\begin{abstract}
Robots capable of hover flight in constrained indoor environments have many applications, however their range is constrained by the high energetic cost of airborne locomotion. Perching allows flying robots to scan their environment without the need to remain aloft. This paper presents the design of a mechanism that allows indoor flying robots to attach to vertical surfaces. To date, solutions that enable flying robot with perching capabilities either require high precision control of the dynamics of the robot, or a mechanism robust to high energy impacts. In this article, we propose a perching mechanism comprising a compliant deployable pad and a passive selfalignment system, that does not require any active control during the attachment procedure. More specifically, a perching mechanism using fibre-based dry adhesives was implemented on a $300 \mathrm{~g}$ flying platform. An adhesive pad was first modeled and optimized in shape for maximum attachment force at the low pre-load forces inherent to hovering platforms. It was then mounted on a deployable mechanism that stays within the structure of the robot during flight and can be deployed when a perching manoeuvre is initiated. Finally, the perching mechanism is integrated onto a real flying robot and successful perching manoeuvres are demonstrated as a proof of concept.
\end{abstract}

\section{INTRODUCTION}

Inspection of buildings by robots is a growing field as this process for exploring collapsed buildings or radioactive areas allows people to take less risk. The advantage of flying robots in this field compared to ground-based robots is that their locomotion does not depend on the topology of the ground. It is thus advantageous to use flying robots in such applications or hybrid robots capable of ground and air locomotion [1], [2], [3]. However, lightweight hovering robots have limited flight time of a few minutes. This paper proposes a perching mechanism to attach on walls providing to flying robots a stable posture for scene surveillance and allowing energy saving.

Very few systems enable flying robots with perching capabilities [4], [5], [6], [7], [8], [9]. These systems either require a high precision control of the dynamics of the robot, or a mechanism robust to high energy impacts. We propose in this article a perching mechanism comprising a compliant deployable pad and a passive self-alignment system, that does not require any active control during the attachment procedure. Our hovering capable platform, that is robust to contacts [10], provides an ideal platform for repeated perching. Robustness to contacts with the environment makes

\footnotetext{
${ }^{1}$ L. Daler, A. Klaptocz, A. Briod and D. Floreano are with the Laboratory of Intelligent Systems (http://lis.epfl.ch) at Swiss Federal Polytechnic in Lausanne (EPFL), CH1015 Lausanne, Switzerland (email: ludovic.daler@epfl.ch).

${ }^{2} \mathrm{M}$. Sitti is with the NanoRobotics Lab (http://nanolab.me.cmu.edu) at Carnegie Mellon University (CMU), Pittsburgh, PA 15213, USA.
}

successful perching more feasible by limiting damage from contacts with walls.

We propose to use dry fibre adhesives as an attachment material for their repeatable adhesion force and their compliance to many different surfaces. An adhesive pad was first modeled and optimized in shape for maximum attachment force at the low pre-load forces inherent to hovering platforms. The pad is designed to roll against the surface during attachment to increase the adhesion force. The pad was then characterized using different pre-load forces to dimension its size and validate its attachment force. A deployable perching mechanism was then designed using this pad. This mechanism stays within the structure of the robot during flight and when a perching manoeuvre is initiated the mechanism can be deployed using a servo-motor. The mechanism is held in place using a cam mechanism which decouples the servo-motor from the pad. The perching strategy is to fly towards a wall and deploy the attachment mechanism just before touching the wall, which can be done with very basic proximity sensors, while the passive selfalignment system adjusts the orientation of the platform with the wall. Finally, we implemented this perching mechanism onto a flying robot and demonstrated perching on the remote controlled platform as a proof of concept.

\section{RELATED WORK}

There are many different attachment mechanisms that could be used to attach to walls and these mechanisms must be adapted to the surface they are used on.

One solution is to use spikes or spines. One small glider perches on walls with the use of small needles [4]. This method is repeatable and works well on soft surfaces, however it does not work on hard surfaces. Lussier et al. [5] presented a glider that uses micro-spines to attach on the walls. The major drawback is that it can not attach on smooth surfaces because of lack of asperities. The advantage of fibre adhesives compared to these solutions is that it can attach on soft and hard materials, as well as on smooth and slightly rough surfaces.

When attaching to ferromagnetic walls or ceilings, one efficient solution is to use magnets to obtain the necessary adhesion strength. One flying robot uses actuated magnets [11] to attach to ferromagnetic surfaces, however many real-world surfaces are not flat and ferromagnetic.

Electro-adhesion could be used for attachment [12]; it offers robust clamping over many surfaces, low power consumption, and has fast and controllable adhesion. However, the surface must be minimally conductive and free of large asperities. 
Dry adhesives are structured surfaces that use Van der Waals force in order to adhere on surfaces. The surface is composed of micro-fibres that maximize the contact area, and thus maximizes the overall Van der Waals force between the two surfaces. Many different groups have used dry adhesives for wall climbing with, legged robots [13], [14], [15], [16], or Mini Whegs robot [17].

We have discussed many different solutions that could be used to perch on walls such as micro-spines, needles, magnets, electro-adhesive, and dry adhesive. All those solutions have advantages and drawbacks, and are developed for different surfaces, which make them difficult to compare. The attachment mechanism should work on a wide variety of surfaces, in order to enable our flying robot to perch on different wall surfaces using a very simple perching strategy. Therefore, fibre adhesive seems to be the most promising technology because it is lightweight, works on many surfaces, does not require energy to maintain the adhesion, and provides repeatable adhesion forces. This paper is the first that proposes to use this technology to achieve perching with a flying robot.

Most attempts at perching flying robots required a good control of the plane in order to perform a specific manoeuvre to attach to a surface. The glider presented by Lussier et $a l$. [5] flies towards walls and detects them with an ultrasonic distance range sensor in order to pitch up at the right time. This method requires high level control, and therefore, reduces the success rate. A glider able to perch on a rope has been developed by Roberts et al. [6]. The glider is controlled off-board using 3D tracking with six cameras. This solution requires a high level of control and external feedback. Moreover, it requires a string to perch, which is a very specific landing site.

Contacting the wall before engaging perching is the solution chosen by Kovac et al. [4] for its simplicity. It is also the approach used by the Sticky-Pad Plane [7] that can perch on vertical walls by the use of glue-pad dispenser placed at the front of a small RC aircraft. We believe that such approach that relies on the smartness of the mechanical design rather than on the control of the perching manoeuvre is more robust and closer to the great perching abilities of insects.

\section{DESIGN AND RESULTS}

This section first presents the adhesive material that is used to attach to smooth surfaces. A layer of foam is used between the pad and the micro-fibres to increase the compliance and the resistance to peel off. Then, a new technique to preload the micro-fibres and increase the adhesion that we can get from low pre-load forces is presented. It consists of a rolling motion of the adhesive pad. Finally, the design of the adhesive pad is presented, which includes sizing and design of the shape of the pad. A solution for self-alignment of the pad to the wall, and the design of an actively deployable mechanism (required to retract the adhesive pad during the flight) are given.
TABLE I

PROPERTIES OF THE FIBRES FOR DIFFERENT LOAD CONFIGURATIONS.

\begin{tabular}{|l|l|}
\hline Load configuration & Measured value \\
\hline Normal peel off & $0.08 \mathrm{~N} / \mathrm{cm}$ \\
Pull & $1 \mathrm{~N} / \mathrm{cm}^{2}$ \\
Shear & $4 \mathrm{~N} / \mathrm{cm}^{2}$ \\
\hline
\end{tabular}

\section{A. Fibre Adhesives' Characteristics}

Many different materials could be used to produce fibre adhesives, for these experiments we used a polyurethane elastomer (ST-1060 A/B) with a hardness of 60 Shore A and a density of $1.05 \mathrm{~g} / \mathrm{cm}^{3}$. Previous research has shown that polyurethane provides high normal and shear adhesion [18], nevertheless polyurethane is not very stable chemically, and changes properties due to oxidation and exposure to UV light. The fibres that we used (Fig. 1) are vertical, have a diameter of $40 \mu \mathrm{m}$ and a height of $100 \mu \mathrm{m}$ with mushroom tips (tips have a diameter of $80 \mu \mathrm{m}$ ). The choice of the geometry of the fibres was done by referencing previous research conducted in the NanoRobotics Laboratory [19]. Table 1 gives the properties of our fibres, measured for different load configurations. The resistance to a tensile force is stronger than the resistance to peel off, which is obvious as the tensile force is distributed over the whole area, whereas the peel off force is applied on a line. The peel off force depends on the rigidity of the backing layer of the fibres sample. A thin and soft backing layer induces a smaller area that sustain the peel off force, which leads to a low resistance to peel off, but allows more compliance to the surface. Conversely, a thick and rigid backing layer will resist a higher peel off force, but will decrease dramatically the adhesion if the surface and the sample are not perfectly flat and parallel.

Therefore, there is a compromise between compliance and resistance to peel off. Experiments that we did showed that the best results are obtained by having a thin backing layer that is compliant, and a layer of foam that distributes the peel off force over a large area (Fig. 1).

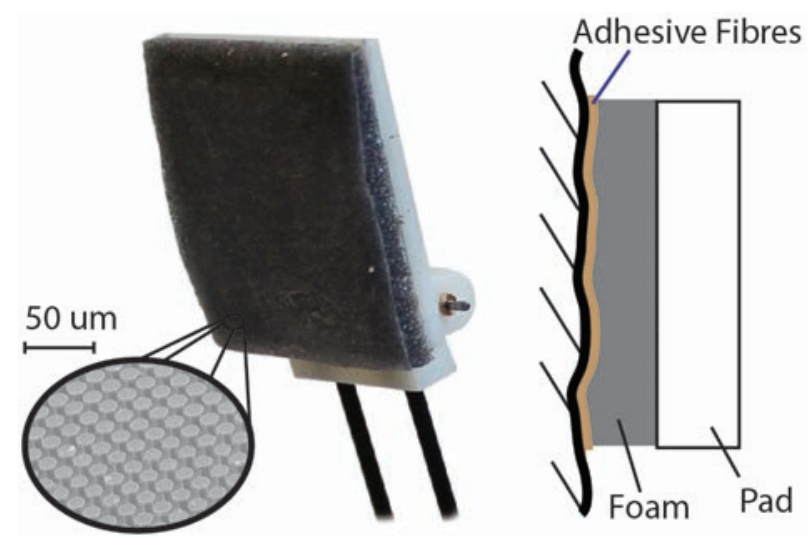

Fig. 1. From left to right: SEM micrograph of the fibre adhesives, adhesive pad with micro-fibres and a layer of foam, and a schematic representation of how the foam increases the compliance of the pad and distributes the peel off force. 


\section{B. Optimization of the Adhesion Force}

In this subsection we will present a new method to increase the adhesion force that can be obtained from adhesive fibres with low pre-load forces. We first characterized the adhesion as a function of the pre-load, thus a load cell was used to measure the adhesion of a small sample of fibres, with a setup allowing measurement of both pre-load and adhesion of the fibres. The sample is made of the fibres presented above with a foam backing layer, as shown in Fig. 1. From the data collected we saw that the relation between the adhesion and the pre-load is an exponential, as:

$$
P_{\text {adhesion }}=P_{\max }\left(1-e^{-\frac{P_{\text {preload }}}{\tau}}\right)\left[N / m^{2}\right],
$$

where $P_{\max }\left(15500 \mathrm{~N} / \mathrm{m}^{2}\right.$, on acrylic) is the maximum adhesion pressure that we can get from the fibres and $\tau$ $\left(3500 \mathrm{~N} / \mathrm{m}^{2}\right.$, on acrylic) is a constant which depends on the properties of the fibres and of the surface.

Now, we can compute the pressure pre-load $P_{\text {Preload }}=$ $F_{\text {Preload }} / A, A$ being the area of the pad. If we use the adhesion equation (Eq. 1) we can compute the adhesion force:

$$
F_{\text {Adhesion }}=A P_{\max }\left(1-e^{-\frac{F_{\text {Preload }}}{A \tau}}\right)[N] .
$$

Finally, we can invert this equation to give the pre-load force required:

$$
F_{\text {Preload }}=\tau A \ln \left(1-\frac{F_{\text {Adhesion }}}{A P_{\max }}\right)[N],
$$

which must be minimized. From this equation we can see that the pre-load force decreases with a large area A (the logarithmic term decreases faster than the proportional term increases), therefore the area must be as large as possible to reduce the required pre-load force. However, for practical reasons we can not have a huge pad on the robot, thus the solution is to minimize $\tau$. A small $\tau$ will give a high adhesion force for a low pre-load force. As mentioned previously this parameter depends on the properties of the fibres. These properties are already optimized, thus to decrease the preload force required, a curved surface was added at the top of the pad, as shown in Fig. 2. The pad first touches the wall with the top of the curved surface and then rolls on this curved surface to end up with the flat surface against the wall. The rolling motion increases the adhesion as we use the adhesion of the first portion in contact to pre-load the next one, and so on. This curved surface follows the shape of a spiral; the curvature decreases linearly between the top of the pad and the flat part of the pad.

In order to optimize the adhesion of this pad, a fully automated adhesion measurement set-up was designed to measure the adhesion in a repeatable way. Two pads of different areas were used and all fibres used for these measurements were manufactured from the same batch. All the experiments were done the same day to avoid perturbations due to temperature, humidity and degradation of the polyurethane elastomer.

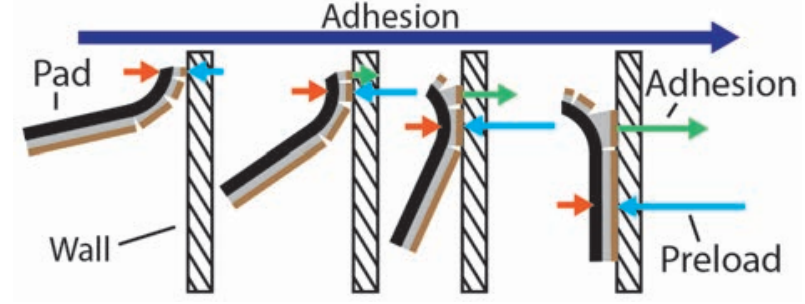

Fig. 2. Pad rolling against the wall. The pad first touches the wall with the top of the curved surface, then the adhesion of this first part combined with the force applied by the robot are used to pre-load the second part, and so on.

Figure 3 shows the results for two different areas of pad; $20 \times 20 \mathrm{~mm}$, and $15 \times 15 \mathrm{~mm}$, each pad was manufactured once with a curved surface at its top and once without it. The solid lines are for the pads without the curved surface and the doted lines for the pads with the curved surface. We took seven times the same measures and plotted the mean and the standard deviation for each point. We measured the adhesion for each of the four pads for pre-loads going from $100 \mathrm{mN}$ to $1200 \mathrm{mN}$ by steps of $100 \mathrm{mN}$.

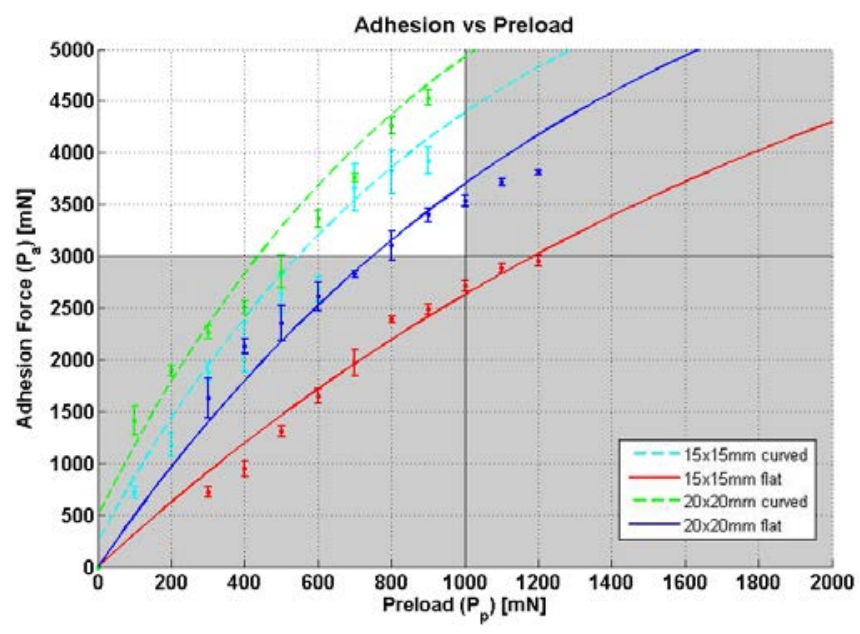

Fig. 3. Adhesion force as function of the pre-load for two different areas of pad. Doted lines are with the curved surface at the top of the pads and the solid lines are without the curved surface.

The adhesion must be higher than the weight that the pad must hold (here we plotted the weight of the robot; $300 \mathrm{~g}$ ) and the pre-load lower than the maximum force that can be applied with the robot. This is given by the thrust of the robot multiplied by the sinus of the angle between the robot and the wall (Fig. 7.4). Thus, the targeted region is the one with the white background. These results show that a flat pad without the curve and of area $20 \times 20 \mathrm{~mm}$ is sufficient to bear the weight of the robot. However, we can see that the adhesion can be increased by 30 percent if we use the same pad with a curved surface at its top for the same pre-load force; $\tau$ is more than twice smaller for the pads with the curved surface. Thus, the curved surface is recommended if we want to have enough margin on the adhesion force for low pre-load forces. 
These results were obtained on an acrylic surface, similar results were obtained on glass (windows) and other very smooth surface. However, on rough surfaces, wet surfaces, or dirty surfaces the adhesion is dramatically reduced.

\section{Design of the Mechanism}

Figure 4 shows the forces exerted on a cylinder representative of any flying platform hanging against a wall. From this diagram we can deduce the shear and tensile forces exerted on the adhesive pad.

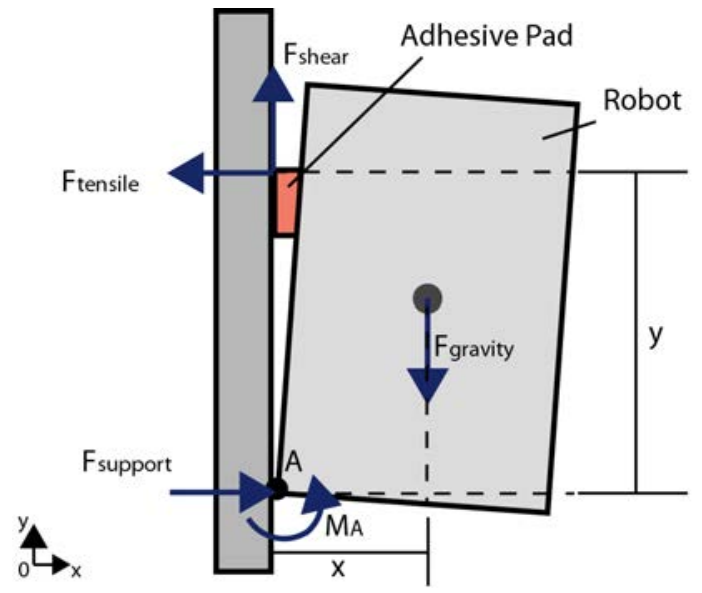

Fig. 4. Representation of the forces applied to a cylindrical robot hanging against a wall.

Using Newton's Second Law and Euler's Second Law around point $\mathrm{A}$, we have:

$$
\begin{aligned}
F_{\text {shear }} & =m g[N] \\
F_{\text {tensile }} & =m g \frac{x}{y}[N]
\end{aligned}
$$

The tensile force is inversely proportional to the distance y between the adhesive pad and the second contact point with the wall $\mathrm{A}$, therefore this distance must be maximized. The best solution to increase this distance is to add an arm to the robot to reach a higher point of contact. If this distance is long enough, mostly shearing will be applied to the adhesive pad. The length of the pad was designed to have the adhesive pad over the center of mass of the robot when it is in the retracted position (Fig. 7.2).

From Fig. 5 we can deduce the maximum stress applied to the fibre adhesives, given by:

$$
\sigma_{\text {max }}=\frac{F_{\text {tensile }}}{A}+\frac{F_{\text {shear }} \cdot d^{2}}{I_{\text {Pad }}}\left[N / m^{2}\right],
$$

where $A$ is the area of the pad, $d$ the distance between the wall and the point of application of the force, and $I_{P a d}$ is the second moment of area. The first term is the stress caused by the tensile force and the second term results from the peeling moment. In order to minimize the second term we have to design the pad in a way that $d$ is the smallest possible and $I_{P a d}$ the largest possible, where $I_{P a d}$ is given by:

$$
I_{P a d}=\frac{w h^{3}}{12}\left[m^{4}\right]
$$

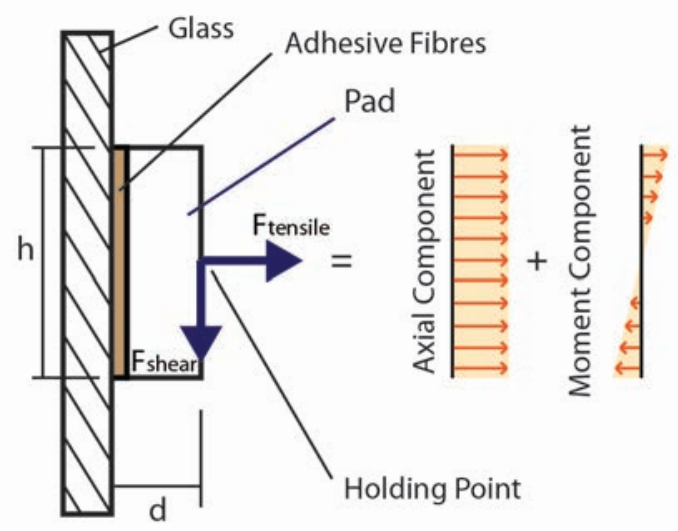

Fig. 5. Loading of the fibre adhesives. The axial component is due to the tensile force and the moment component is due to the shear force.

Where $w$ and $h$ are the width and height of the sample. From this equation we can deduce that the pad should be thin and that the height should be greater than the width. Figure 7 shows the pad of dimensions $18 \times 22 \times 2 \mathrm{~mm}$ with the curved surface at its top.

A main issue to consider for the design of the perching mechanism is its stiffness. If the mechanism is soft, it provides good compliance to the surface but it is not possible to apply enough pressure to pre-load the fibres. Conversely, a stiff mechanism is good for pre-loading the fibres but not for compliance. The optimal stiffness must be found for the mechanism that gives enough compliance to counteract misalignment between the robot and the wall, and at the same time, allows for enough pre-loading of the adhesive pad to obtain high adhesion. In this paper we propose a mechanism that connects the pad to the robot by two carbon fibre rods. The flexibility of the two carbon rods allows the pad to conform to the surface. The deflection $f$ of a rod of length 1 due to a perpendicular force $\mathrm{F}$ at it extremity is given by:

$$
f=\frac{F l^{3}}{3 E I_{\text {Rod }}}[m]
$$

where $\mathrm{E}$ is the Young modulus (150 GPa for a carbon fibre rod), and $I_{R o d}$ is given by:

$$
I_{\text {Rod }}=\frac{\pi r^{4}}{4}\left[m^{4}\right] .
$$

From these equations we can deduce the radius of the rods:

$$
r=\sqrt[4]{\frac{4 F l^{3}}{3 \pi E f}}[m] .
$$

For a deflection of the rods of $10 \mathrm{~mm}$ (which is reasonable according to the size of the pad) it gives a radius of $1 \mathrm{~mm}$ for the two rods.

For the deployment of the pad, we investigated two different approaches, we can link the pad directly to an actuator or actuate it indirectly. The inconvenience of direct actuation is that all the impact forces applied on the pad, during a 
collision with a wall, are going through its motor. The motor will either move, which would give too much compliance to the mechanism, or break. Therefore, we decided to use an indirect actuation to protect the motor. The motor allows the system to go from one stable position to the other. We use a cam that is actuated by a servo-motor, to push against the arm of the pad. Figure 6 shows the cam, that has two flat surfaces, in its two stable positions 1 (pad retracted) and 2 (pad deployed). The use of the cam in the design prevents any force on the pad from back-driving the servo-motor, since in these positions the force applied by the arm on the cam is in the direction of the axe of rotation of the cam.

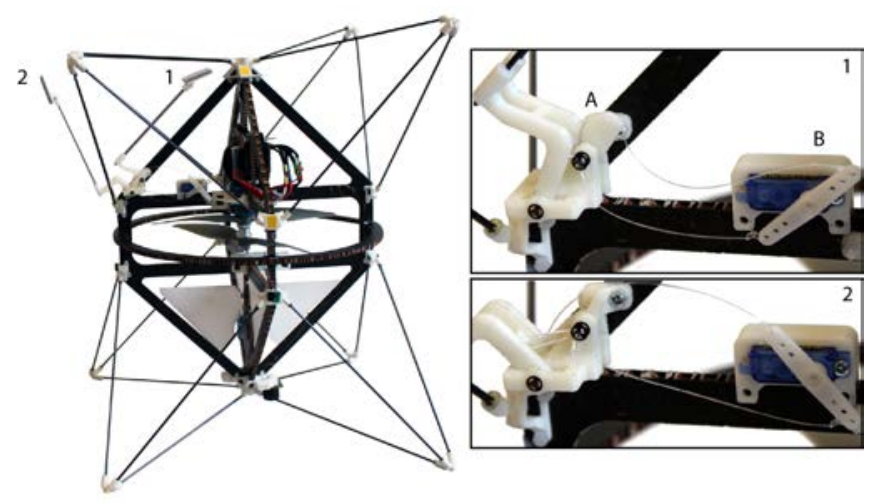

Fig. 6. Final design of the deployable mechanism on the robot. A) Cam mechanism. B) Servo-motor. 1) Pad retracted position. 2) Pad deployed position.

Figure 7.4 shows how the robot aligns with the wall thanks to a smart passive self-alignment system. The eight Euler springs in a tetrahedral configuration around the robot were originally designed to elastically absorb the energy of an impact [10]. We used this existing structure to self-align the robot with the walls and designed the arm of the perching mechanism in a way that the top of the pad touches the wall on the line joining the tips of two tetrahedrons next to it. Thus, this mechanism does not require the use of sensors to align the platform with the wall before perching.

\section{VALIDATION}

We used the final prototype presented in Fig. 7 to implement a perching behaviour. This figure shows a perching sequence demonstrating the perching abilities of the robot. We start with the robot on the ground, take off in the middle of the room, fly around with the help of the IMU stabilization, and can bump into walls thanks to the protective structure [10]. When a perching manoeuvre is initiated by the pilot we first deploy the arm with the adhesive pad, and fly directly towards a wall, the robot contacts the wall with the top of its adhesive pad. The engines' thrust is slowly reduced until the robot hangs against the wall, held only by its adhesive pad. Once the pad is properly in contact with the wall it can stay attached as long as needed since the adhesion does not decrease with time.

We performed a successful remote controlled experiment as a proof of concept showing how the robot can attach using the perching mechanism, the pad attaches as planned and the robot passively self-aligns with the wall. The approximate positioning of the robot towards the wall is reached by the pilot, however this task could be realized by the use distance sensors on each of the four sides of the robot. The use of distance sensors, force sensors in the pad, and speed estimation of the robot would make the system more robust and repeatable. Statistics about percentage of successful perching can not be given since it depends on the skills of the pilot. However, these results validate the concepts presented in the previous section and future work will investigate the success rate with respect to several parameters like angle of approach, approach speed, type of wall, and attachment repeatability with a single pad. A sample of flight and attachments can be seen in the accompanying video. Future work will also be done on detachment; a simple peeling mechanism could be used.

\section{CONCLUSIONS}

We designed and tested on a real flying platform a perching mechanism that requires very little active control. The adhesive pad can be deployed just before perching on a wall, and is well protected inside the robot when the robot is flying or hovering. This mechanism consumes little energy; we use the servo-motor to switch the mechanism from one passively stable position to another. Once the cam is in one of the two passively stable positions the mechanism does not consume any energy. Thanks to the passive selfalignment system and to the compliance of the arm of the pad, the perching manoeuvre is very simple and repeatable. In addition, we optimized the shape and the size of the pad to maximize the adhesion that we can get from dry fibre adhesives with low pre-load forces. Finally, we demonstrated successful perching manoeuvres with the flying platform, which can be seen in the accompanying video.

\section{ACKNOWLEDGMENTS}

This work was supported by armasuisse, competence sector Science + Technology for the Swiss Federal Department of Defense, Civil Protection and Sports, and by the Swiss National Science Foundation through the National Centre of Competence in Research Robotics.

\section{REFERENCES}

[1] K. Peterson and R. Fearing, "Experimental dynamics of wing assisted running for a bipedal ornithopter," in Intelligent Robots and Systems (IROS), 2011 IEEE/RSJ International Conference on. IEEE, 2011, pp. 5080-5086.

[2] R. Bachmann, F. Boria, R. Vaidyanathan, P. Ifju, and R. Quinn, "A biologically inspired micro-vehicle capable of aerial and terrestrial locomotion," Mechanism and Machine Theory, vol. 44, no. 3, pp. 513526, 2009.

[3] M. Itasse and J. Moschettai, "Equilibrium transition study for a hybrid mav," in International Micro Air Vehicle Conference and Flight Competition, 2011 IMAV, oct. 2011.

[4] M. Kovac, J. Germann, C. Hurzeler, R. Siegwart, and D. Floreano, "A Perching Mechanism for Micro Aerial Vehicles," Journal of MicroNano Mechatronics, pp. 77-91, 2010.

[5] A. Lussier Desbiens, A. Asbeck, and M. Cutkosky, "Landing, perching and taking off from vertical surfaces," The International Journal of Robotics Research, vol. 30, no. 3, p. 355, 2011. 


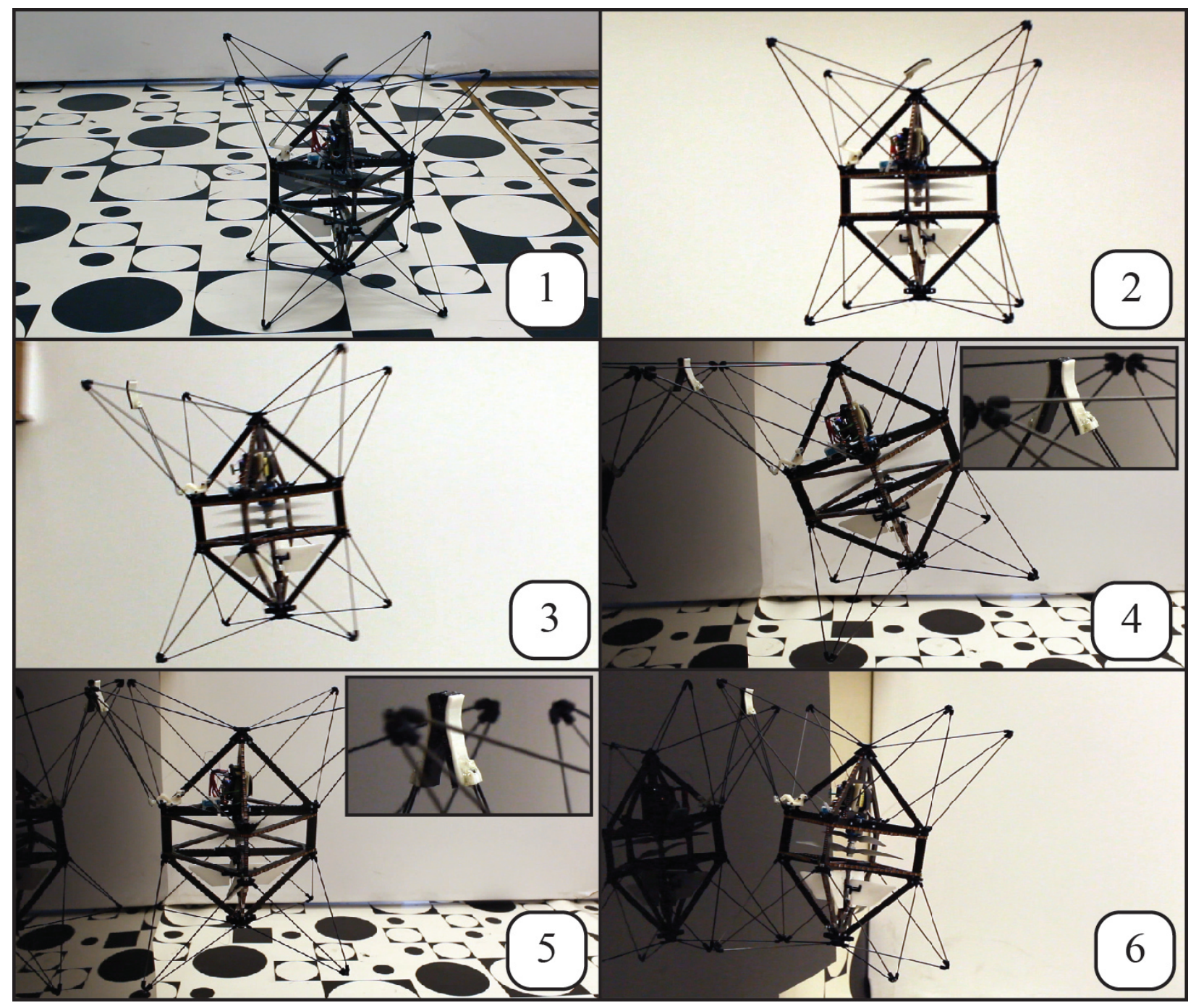

Fig. 7. Sequence of the perching manoeuvre. 1) The robot takes off in the middle of the room. 2) The robot flies thanks to its IMU stabilization, and it can bump into walls thanks to its protective structure. 3) The adhesive pad is deployed. 4) The robot contacts the wall with the top of its adhesive pad. 5) The engines' thrust is slowly reduced. 6) The robot is hanging against the wall, held only by its adhesive pad.

[6] J. Roberts, R. Cory, and R. Tedrake, "On the controllability of fixedwing perching," in American Controls Conference (ACC), 2009.

[7] M. Anderson, C. Perry, B. Hua, D. Olsen, J. Parcus, K. Pederson, and D. Jensen, "The sticky-pad plane and other innovative concepts for perching uavs," in 47 th AIAA Aerospace Sciences Meeting, 2009.

[8] D. Mellinger, M. Shomin, and V. Kumar, "Control of quadrotors for robust perching and landing," in Proceedings of International Powered Lift Conference,(Philadelphia, PA), vol. 1, 2010.

[9] C. Doyle, J. Bird, T. Isom, C. Johnson, J. Kallman, J. Simpson, R. King, J. Abbott, and M. Minor, "Avian-inspired passive perching mechanism for robotic rotorcraft," in Intelligent Robots and Systems (IROS), 2011 IEEE/RSJ International Conference on. IEEE, 2011, pp. 4975-4980.

[10] A. Klaptocz, A. Briod, L. Daler, J.-C. Zufferey, and D. Floreano, "Elastic collision protection for flying robots using euler springs," IEEE Transactions on Mechatronics, p. In review, 2012.

[11] J. Roberts, J. Zufferey, and D. Floreano, "Energy management for indoor hovering robots," in IEEE/RSJ International Conference on Intelligent Robots and Systems (IROS2008), 2008, pp. 1242-1247.

[12] H. Prahlad, R. Pelrine, S. Stanford, J. Marlow, and R. Kornbluh, "Electroadhesive robotswall climbing robots enabled by a novel, robust, and electrically controllable adhesion technology," in Robotics and Automation, 2008. ICRA 2008. IEEE International Conference on. IEEE, 2008, pp. 3028-3033.

[13] S. Kim, M. Spenko, S. Trujillo, B. Heyneman, V. Mattoli, and
M. Cutkosky, "Whole body adhesion: hierarchical, directional and distributed control of adhesive forces for a climbing robot," in 2007 IEEE International Conference on Robotics and Automation, 2007, pp. $1268-1273$.

[14] M. Spenko, G. Haynes, J. Saunders, M. Cutkosky, A. Rizzi, et al., "Biologically inspired climbing with a hexapedal robot," Journal of Field Robotics, vol. 25, no. 4-5, pp. 223-242, 2008.

[15] A. Asbeck, S. Dastoor, A. Parness, L. Fullerton, N. Esparza, D. Soto, B. Heyneman, and M. Cutkosky, "Climbing rough vertical surfaces with hierarchical directional adhesion," in Robotics and Automation, 2009. ICRA'09. IEEE International Conference on. IEEE, 2009, pp. 2675-2680.

[16] M. Murphy, C. Kute, Y. Menguc, and M. Sitti, "Waalbot II: Adhesion Recovery and Improved Performance of a Climbing Robot using Fibrillar Adhesives," The International Journal of Robotics Research, vol. 30, no. 1, p. 118, 2011.

[17] K. Daltorio, T. Wei, A. Horchler, L. Southard, G. Wile, R. Quinn, S. Gorb, and R. Ritzmann, "Mini-Whegs TM Climbs Steep Surfaces Using Insect-inspired Attachment Mechanisms," The International Journal of Robotics Research, vol. 28, no. 2, p. 285, 2009.

[18] B. Aksak, M. Murphy, and M. Sitti, "Adhesion of biologically inspired vertical and angled polymer microfiber arrays," Langmuir, vol. 23, no. 6, pp. 3322-3332, 2007.

[19] S. Kim, "Polymer micro/nanofiber arrays with mushroom-shaped tips as gecko foot-hairs inspired adhesives," 2009. 